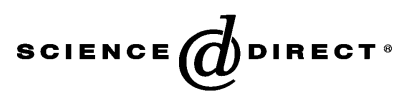

European Polymer Journal 42 (2006) 1953
EUROPEAN

POLYMER

JOURNAL

www.elsevier.com/locate/europolj

Preface

\title{
Macromolecular Nanotechnology in European Polymer Journal
}

Earlier this year we announced [1] that European Polymer Journal (EPJ) will launch a new section focusing on Macromolecular Nanotechnology. "...This section will encompass original papers (communications and full articles) focusing on fundamental research, nanoscale technologies, nano-structured polymeric materials, and biological nanosystems, as well as review articles (usually upon invitation by the editors) reflecting major achievements and trends in macromolecular science and technology at the nanoscale." We have a broad definition for nanoscience and nanotechnology, which is described in detail in [1]. We are very pleased and excited to publish the first set of nine articles on Macromolecular Nanotechnology in this issue of EPJ.

Chemical synthesis of controlled nanoscale (composite) structures featuring polymers and hybrid nanosized materials has received attention in several submissions. Nanofabrication with special processing techniques, such as electrospinning and soft lithography (the latter for biological applications) have been used to prepare nanoscale functional platforms and structures across the length scales from nano- to micro-metres. Self-organization in microphase separated block copolymers, and alignment effects on the nanoscale using external fields are discussed in several contributions to obtain functional nanostructures. There are also examples for the use of advanced scanning probe microscopies like AFM to characterize the nanostructures. Achieving significant improvement of mechanical performance and enhancement of the function of nanostructured composites motivated two studies. Preparation of platforms for scale-dependent biological applications (biosensors, diagnostic arrays, cell adhesion), electroactive materials for devices, separation technology, and structural materials are also among the application targets of several articles.

In our opinion this selection gives the reader a representative first impression of the quality, breadth and depth, and the plethora of advanced applications, which have attracted tremendous recent interest in nanoscience and nanotechnology of macromolecular substances. These are representative of articles to be published in this new section. We hope you find this useful and invite you to submit your papers to us for consideration. EPJ has been working hard to improve the quality of our Journal as testified by the increase in impact factor and will be processing your articles as efficiently as possible.

Enjoy!

\section{Reference}

[1] Vancso GJ. Eur Polym J 2006;42(1):1.

G. Julius Vancso

University of Twente, Department of Materials,

Science and Technology of Polymers and $\mathrm{MESA}^{+}$Institute for Nanotechnology, P.O. Box 217, 7500 Enschede, Netherlands

E-mail address: g.j.vancso@tnw.utwente.nl

Available online 26 July 2006 